\section{Discrete foci containing RNase $A$ are found in nucleoli of HeLa cells aged in culture}

\author{
M. Costanzo, ${ }^{1}$ B. Cisterna, ${ }^{1}$ \\ O.O. Zharskaya, ${ }^{2}$ O.V. Zatsepina, ${ }^{2}$ \\ M. Biggiogera ${ }^{1}$
}

'Laboratorio di Biologia Cellulare e

Neurobiologia, Dipartimento di Biologia

Animale, Università di Pavia, Italy;

2Shemyakin-Ovchinnikov Institute of

Bioorganic Chemistry, Russian Academy

of Sciences, Moscow, Russia

\begin{abstract}
We have studied by means of ultrastructural immunocytochemistry the localization of RNase A in nuclei of HeLa cells in control conditions and following cell ageing in culture. We have found that roundish, electron dense foci, which contain a significant amount of RNase A, can be detected within nucleoli of aged cells. These bodies also contain RNA and lack ribosomal S3 proteins, and may represent either simple storage sites or areas where RNA degradation takes place.
\end{abstract}

\section{Introduction}

The cell nucleus contains several different structures or functional domains, among which the nucleolus, the interchromatin granule clusters (IGC), Cajal bodies and other nuclear bodies, whose functions range from the storage of specific factors to the shuttling of proteins among the various domains. They can be found in several locations in the cell nucleus; in many cases, the functions of these bodies are still to be completely elucidated. For some of these structures, such as promyelocytic leukaemia (PML) bodies or Cajal bodies, marker proteins have been described., ${ }^{2,3}$

In this view, we are studying the presence of several proteins as possible markers of nuclear structures (manuscript in preparation); in particular, we are interested in possible storage structures for nucleases. In fact, as for RNAs, data in the literature suggest that more than $90 \%$ of the newly synthesized RNA is degraded in the nucleus ${ }^{4}$ and this destruction is carried out by means of several mechanisms apt to recognize damaged or nonsense RNA. The reason of such nonproductive RNA synthesis is not clear: one hypothesis claims that this high hnRNA transcription rate would maintain chromatin domains open to prevent the forma- tion of inactive chromatin states. ${ }^{4}$ There is no hint, so far, as to where RNA degradation could take place in the nucleus. It is known that the perichromatin area is the site where transcription, co-transcriptional splicing and cleavage mechanism are located; 5 in this view, it is possible that RNA degradation could be cotranscriptional or immediately subsequent to RNA synthesis. It is also well known that in mammals the most actively synthesised type of RNA is ribosomal RNA (rRNA). ${ }^{6}$ Therefore, we focused our attention on the possible localization of nucleases inside the nucleolus; to this purpose, we have studied by means of ultrastructural immunocytochemistry the nucleolar localization of RNase A in control and aging HeLa cells. Since nuclear degradation of RNA can be accomplished - at least in part - by RNase A, we have used an anti-RNase A antibody to detect this enzyme in the cell nucleus, with the aim of finding whether any storage or shuttling system do exist.

\section{Materials and Methods}

\section{Cells and treatments}

HeLa cells were grown in DMEM medium supplemented with $10 \%$ fetal bovine serum, 2 $\mathrm{mM}$ glutamine and 100 units each of streptomycin and penicillin, in $25 \mathrm{~cm}^{2}$ plastic flasks or on $4 \mathrm{~cm}^{2}$ glass coverslips in multi-well dishes on a air atmosphere containing $5 \%$ of $\mathrm{CO}_{2}$ (all reagents and plastic were from Euroclone, Milan, Italy). The HeLa-Ibch cells represent a subline isolated at the Institute of Bioorganic Chemistry, and are aged cells obtained after six month of culture under the same conditions. These cells were free from any detectable contaminations and were characterized by an increased number of apoptotic cells, which were not considered in the present investigation.

\section{Electron microscopy and ultra- structural immunocytochemistry}

The cells were harvested by trypsinization (0.25\% trypsin in PBS containing 0.5\% EDTA) and immediately fixed with $4 \%$ paraformaldehyde $\left(2 \mathrm{~h}\right.$ at $\left.4^{\circ} \mathrm{C}\right)$ in the culture medium. The cell pellets were embedded in $2 \%$ agar in water, thoroughly rinsed with Sörensen buffer $(\mathrm{pH}$ 7.2) and dehydrated in ethanol. Finally the cells were embedded in LR White resin and polymerized at $60^{\circ} \mathrm{C}$ for $24 \mathrm{~h}$. Thin sections were collected on nickel grids covered with a Formvar-carbon film and incubated on normal goat serum (NGS) for $3 \mathrm{~min}$. For immunolabeling, the grids were floated for $17 \mathrm{~h}$ at $4^{\circ} \mathrm{C}$ onto a solution of $0.1 \%$ bovine serum albumine and
Correspondence: Prof. Marco Biggiogera, Laboratorio di Biologia Cellulare e Neurobiologia, Dipartimento di Biologia Animale, Università di Pavia, via A. Ferrata, 927100 Pavia, Italy.

Tel. +39.0382.986322.

E-mail: marco.biggiogera@unipv.it

Key words: nucleolus, RNase, electron microscopy, immunocytochemistry.

Acknowledgements: the study was in part supported by the CNR-RAS Scientific collaborative project and by the Russian Foundation for Basic Research (grant 08-04-00854). This work was supported by the University of Pavia (Fondo di Ateneo per la Ricerca 2010).

The authors are grateful to Drs. Carlhoun and Fornasiero for critical reading of the manuscript.

Received for publication: 4 April 2011. Accepted for publication: 11 April 2011.

This work is licensed under a Creative Commons Attribution 3.0 License (by-nc 3.0).

(C) Copyright M. Costanzo et al., 2011

Licensee PAGEPress, Italy

European Journal of Histochemistry 2011; 55:e15 doi:10.4081/ejh.2011.e15

0.05\% Tween 20 in PBS containing the primary anti-RNase A antibody diluted 1:200. After rinsing with PBS-Tween and PBS, grids were incubated with NGS as above and then reacted with a goat-anti rabbit secondary antibody conjugated with $12 \mathrm{~nm}$ or $6 \mathrm{~nm}$ colloidal gold (The Jackson Lab., Bar Harbor, ME, USA) for $30 \mathrm{~min}$ at room temperature (RT), rinsed and finally stained. To reduce chromatin contrast and selectively reveal nuclear ribonucleoprotein constituents, the sections were bleached by the EDTA method ${ }^{8}$ or selectively stained for RNA with terbium citrate. ${ }^{9}$

The samples were observed on a Zeiss 900 EM operating at $80 \mathrm{kV}$. The gold grains in some of the micrographs have been digitally contrasted with Photoshop software.

\section{Immunofluorescence}

HeLa cells were grown on coverslips, fixed for 7 min with 1\% paraformaldehyde in PBS, rinsed in PBS and left 30 min in 70\% ethanol at $-20^{\circ} \mathrm{C}$.

The coverslips were rehydrated in PBS and then incubated with the anti-RNase A antibody, diluted 1:150 in PBS for 1h at RT, rinsed in PBS and incubated with an Alexa 488-conjugated goat-anti rabbit antibody, diluted 1:200 for $1 \mathrm{~h}$ at RT, rinsed, counterstained with Hoechst 33342 for 5 min, and finally mounted with Mowiol. The cells were observed with an Olympus BX51 microscope equipped with a 100 W mercury lamp under the following conditions: 330 - to 385 -nm excitation filter (excf), 
400-nm dichroic mirror (dm), and 420-nm barrier filter (bf), for Hoechst 33258; 450- to 480$\mathrm{nm}$ excf, $500-\mathrm{nm} \mathrm{dm}$, and $515 \mathrm{~nm}$ bf for Alexa 488. Images were recorded with an Olympus Camedia C-5050 digital camera and stored on a PC by the Olympus software for processing and printing.

\section{Results}

The anti-RNase A antibody used in this study recognizes the enzyme in several mammalian species, including humans. ${ }^{7}$ Normally, a great part of the labelling is present in the cytoplasm (not shown) as well as in specific subnuclear locations such as the perichromatin area (manuscript in preparation).

Intriguingly, in aging HeLa cells the antibody often detects the enzyme also in discrete parts of the nucleolus, and the labelling always covers roughly roundish areas about 0.2-0.4 $\mu \mathrm{m}$ in diameter (Figure 1a) which are more electrondense than the nucleolar body. This area is finely fibrillar and homogeneous and is morphologically different from any of the typical nucleolar components, the dense fibrillar component, the fibrilar centre or the granular component. After terbium staining to specifically reveal RNA, an area similar to that labelled in Figure la can be seen as containing RNA fibrils (Figure 1b and inset). The terbium staining confirmed that this RNA-containing structure does not seem to co-locate with any of the nucleolar components. Apparently, there is no preferential relationship with a given nucleolar component. After immunofluorescence for RNase A detection and DNA counterstaining, the nuclear labelling was mainly found in the nucleolus, where RNase A distribution proved to be inhomogeneous with some more densely labelled areas (arrow in Figure 1c). This seems consistent with the RNase A focal localization observed at electron microscopy. The foci described above are clearly revealed by anti-RNase A immunolabelling and terbium staining (Figure 1d,f). In some cases, labelling for the nucleolar protein S3, present in the small ribosomal subunit, can be seen in the nucleolar body at the periphery of one of the terbium-positive nucleolar foci (Figure 1e). These features were never found in not aging control HeLa cells (not shown).

\section{Discussion}

The nucleolus is the site of pre-ribosome biogenesis and is also involved in other, noncanonical, functions. ${ }^{6,10}$ The main activity, however, is the synthesis of rRNAs and its association with ribosomal proteins to form the ribosomal subunits. Transcriptional activity is very active in HeLa cells, and it is known to decrease with aging in culture.

Interestingly, in the nucleolar body of aging HeLa cells we have found discrete, focal regions, which are enriched in RNase A. Since the antibody used recognizes both the active and the inactive form of the enzyme, it is impossible to know whether the foci represent simple storage sites or areas where RNA degradation takes place. However, since these bodies always contain detectable amounts of RNA, as seen with terbium staining, RNase A is very probably inactive. The presence of these structures is not constant, and their morpholo-
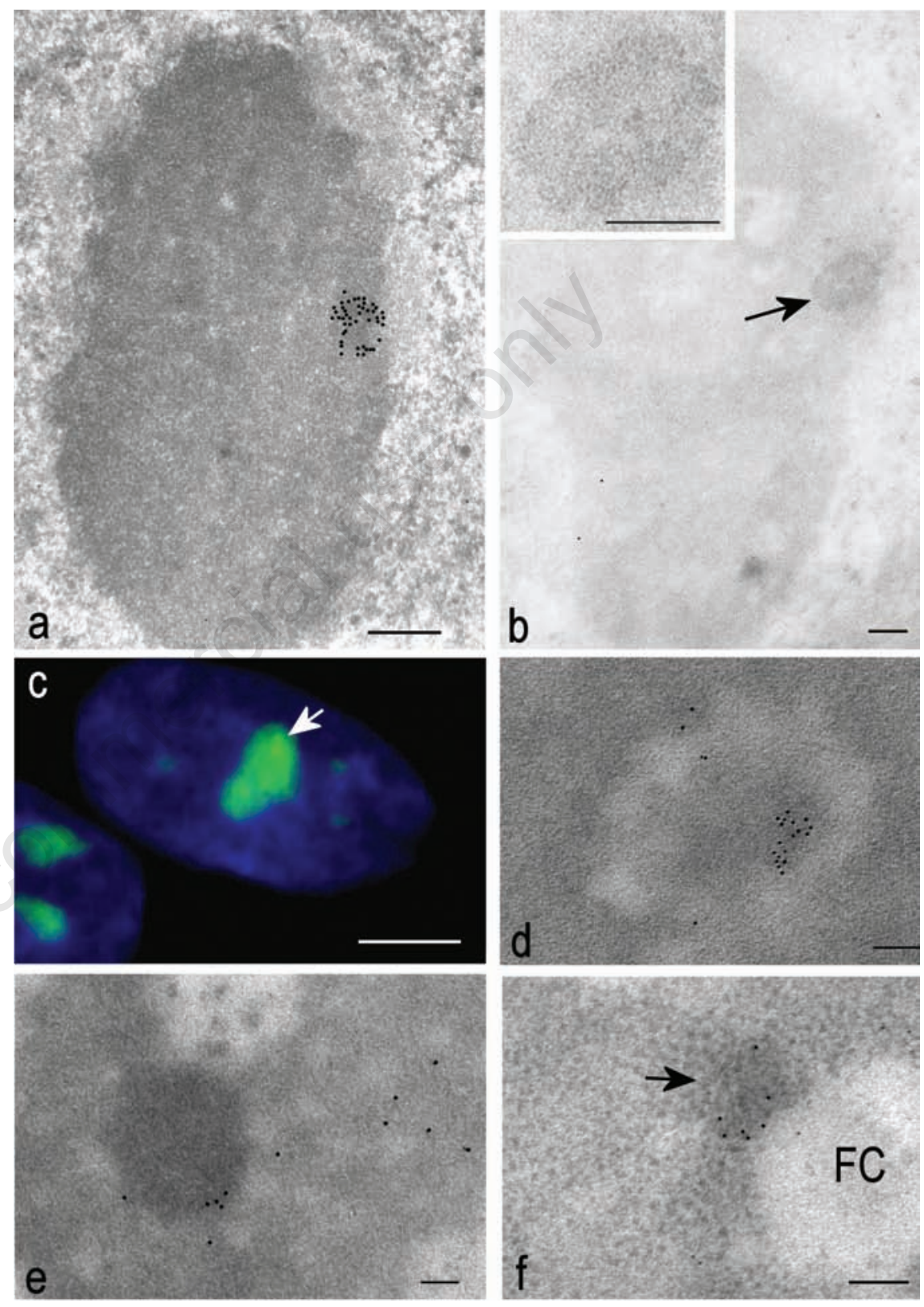

Figure 1. a) Aged HeLa cell after EDTA staining. The labelling for RNAase A is present exclusively over a dense area near the periphery of the nucleolus. Bar: $0.25 \mu \mathrm{m}$. b) Aged HeLa cell after terbium staining specific for RNA. The peripheral roundish area (arrow) contains RNA fibers. In the inset, the fibrils are clearly visible. Bar: $0.25 \mu \mathrm{m}$. c) After immunofluorescence labeling for RNase A, the nucleolus is clearly not homogeneously labelled. The arrow points to a bright spot. Bar: $10 \mu \mathrm{m}$. d) Terbium staining and labelling for RNAse demonstrate the colocalization of these components in the dense structure. Bar: $0.25 \mu \mathrm{m}$. e) The dense body is labelled only at the periphery by the anti-S3 antibody. The ribosomal protein is present in the DFC e GC. Bar: $0.25 \mu \mathrm{m}$. f) Terbium staining and labelling for RNAse A. A dense body (arrow) in the close vicinity of a fibrillar centre (FC). Bar: $0.25 \mu \mathrm{m}$. 
gy is clearly recognizable from the other nucleolar components.

To our knowledge there is no mention of similar structures in the literature. When other nuclear bodies like Cajal bodies were found embedded into the nucleolus, their morphology was still recognizable. ${ }^{11,12}$ Moreover, these foci are different also from the nucleolus-associated domains described by Malatesta et al. ${ }^{13}$ in tissues from hibernating mammals.

An interesting hypothesis would be that RNA degradation takes place near the site of transcription and especially increases whenever the rate of error is particularly high (as it likely occurs in ageing $)^{14,15}$ or there is a need for rapidly blocking the transcript from reaching the cytoplasm: under these circumstances, a particular structure accumulating RNase appears inside the nucleolus. It will be interesting to investigate whether this RNase-containing bodies or foci do also occur in other cell models in vitro after treatments inducing massive impairment of transcription or translation, or in vivo in aging animals.

\section{References}

1. Matera AG, Izaguire-Sierra M, Praveen K, Rajendra TK. Nuclear bodies: random aggregates of sticky proteins or crucibles of macromolecular assembly? Dev Cell. 2009;17:639-47.

2. Raska I, Andrade LE, Ochs RL, Chan EK, Chang CM, Roos G, et al. Immunological and ultrastructural studies of the nuclear coiled body with autoimmune antibodies. Exp Cell Res 1991;195:27-37.

3. Van Damme E, Laukens K, Dang TH, Van Ostade X. A manually curated network of the PML nuclear body interactome reveals an important role for PML-NBs in SUMOylation dynamics. Int $\mathrm{J}$ Biol Sci. 2010:6:51-67.

4. Jackson DA, Pombo A, Iborra FJ. The balance sheet for transcription: an analysis of nuclear RNA metabolism in mammalian cells. Faseb J 2000;14:242-54.

5. Fakan S. Perichromatin fibrils are in situ forms of nascent transcript. Trends Cell Biol 1994;4:86-90.

6. Boulon S, Westman BJ, Hutten S, Boisvert FM, Lamond AI. The nucleolus under stress. Mol Cell. 2010;40:216-27.

7. Painter RG, Tokuyasu KT, Singer SJ. Immunoferritin localization of intracellular antigens: the use of ultracryotomy to obtain ultrathin sections suitable for direct immunoferritin staining. Proc Nat Acad Sci USA 1973;70:1649-53.

8. Bernhard W. A new staining procedure for electron microscopical cytology. J Ultrastruct Res 1969;27:250-65.
9. Biggiogera M, Fakan S. Fine structural specific visualization of RNA on ultrathin sections. J Histochem Cytochem 1998;46: 389-95.

10. Cisterna B, Biggiogera M. Ribosome biogenesis: from structure to dynamics. Int Rev Cell Mol Biol 2010;284:67-111.

11. Malatesta M, Zancanaro C, Martin TE, Chan EKL, Amalric F, Lührmann R,et al. Is the coiled body involved in nucleolar functions? Exp Cell Res 1994; 211:415-9.

12. Dundr M, Misteli T. Biogenesis of nuclear bodies. Cold Spring Harb Perspect Biol. 2010;2:a000711.

13. Malatesta M, Zancanaro C, Biggiogera $\mathrm{M}$. Immunoelectron microscopic characterization of nucleolus-associated domains during hibernation. Microsc Res Tech 2011;74:47-53.

14. Malatesta M, Perdoni F, Muller S, Zancanaro C, Pellicciari C. Nuclei of aged myofibres undergo structural and functional changes suggesting impairment in RNA processing. Eur J Histochem 2009;53: 97-106.

15. Malatesta M, Biggiogera M, Cisterna B, Balietti M, Bertoni-Freddari C, Fattoretti P. Perichromatin fibrils accumulation in hepatocyte nuclei reveals alterations of pre-mRNA processing during aging. DNA Cell Biol 2010;29:49-57. 